\title{
Los factores de resiliencia ante las situaciones traumá- ticas. Análisis tras los atentados del 11 de marzo en una muestra de pacientes en el CSM de Alcalá de Henares.
}

The resilience factors in traumatic situations. Analysis after the terrorist assault in a sample of patients in the mental health services of Alcalá de Henares.

Adolfo Benito Ruiz a ,Laila Oudda Santos b , Guillermo Benito Ruiz ${ }^{\text {, }}$, Guillermo Lahera Forteza ${ }^{\text {, }}$ Alberto Fernández Liria ${ }^{\mathrm{e}}$

${ }^{a}$ Psiquiatra Hospital Virgen de la Misericordia. Toledo. ${ }^{b}$ Médico de Urgencia Hospitalaria. Hospital General de Guadalajara. ${ }^{C}$ Psicólogo. ${ }^{d}$ Psiquiatra, CSM de Torrejón de Ardoz, ${ }^{e}$ Psiquiatra coordinador de área 3 de la CAM.

Correspondencia: Adolfo Benito Ruiz (taurocatapsia@yahoo.com)

Recibido: 3/12/2009; aceptado con modificaciones: 8/03/2010

RESUMEN: Introducción: Los atentados del 11M del 2004 en Madrid supusieron un reto para la sanidad madrileña, debido al gran número de afectados y víctimas. Esta demanda fue especialmente visible en el CSM de Alcalá de Henares, que tuvo que hacer frente a gran número de afectados. El objetivo del estudio es conocer a los dos años y medio de los atentados los factores que los pacientes consideran más les han ayudado a la superación de una situación traumática (de resiliencia).

Material y Método: Se recogió la población de pacientes que acudieron al CSM con un motivo de consulta en relación a los atentados terroristas, mayores de edad con un periodo de inclusión de 3 meses y medio tras los atentados. La muestra final estaba conformada por 104 pacientes, se contactó entre los meses de febrero y marzo del 2007 telefónicamente para que respondiese a 5 cuestiones entre la que se encontraba la relativa a los factores de resiliencia. Las respuestas se transcribieron literalmente y posteriormente se elaboraron una serie de etiquetas o categorías que englobasen a todas las respuestas.

Resultados: De los 104 pacientes se logro contactar con 78 y de ellos 76 accedieron a contestar. El factor de resiliencia más mencionado fue el asociado al entorno sociofamiliar, seguido del tratamiento y en tercer lugar la respuesta individual al trauma y las características propias del individuo, otros factores de resiliencia fueron mencionadas en menor medida. Conclusiones: Nuestros hallazgos resaltan la importancia de el entorno sociofamiliar como el factor de mayor utilidad para la readaptación tras una vivencia traumática, coincidiendo con la mayoría de los estudios. Destaca que las variables asociadas al tratamiento son mencionados solo por 10 pacientes de la muestra, lo que parece indicar una mayor im-
ABSTRACT: Introduction: The 11th of March bomb attack in Madrid suponed a challenge for the madrilenian health sistem, due to the great number of affected and victims. This demand was specifically visible in the Mental Health Services of Alcalá de Henares, that by his geographic situation it had to do in front of great number of affected. The aim of this study is to know 2,5 years after the attacks the factors that the patients consider more have helped for overcoming this traumatic situation (resilience factors).

Material and Method: The sample was conformed for the patients that went to the Mental Health Services with a reason for consultation in relation to the terrorist attack, of legal age with a period of inclusion of 3 months and a half after the attacks. The final sample was conformed by 104 patients which was contacted by phone between the months of February and March of 2007 so tha they responded to 5 questions between it was regarding the resilience factors. The answers were transcribed literally and afterwards was elaborated a series of labels or categories that included all the answers.

Results: 78 of the 104 patients of the sample were contacted of whom 76 acceded to answer. The resilience factor more mentioned was the sociofamiliar factor followed of the treatment factor and thirdly the individual response to the trauma and the own characteristics of the individual, other resilience factors were mentioned to a lesser extend.

Conclusions: Our findings emphasizes the importance of the sociofamiliar factors as the factors of greater utility for the readjustment after a traumatic experience, as the majority of the studies agrees. It is remarkable that the variables associated with the treatment although are the second factor of valued 
portancia del apoyo social para la superación de una vivencia traumática y una menor utilidad para los pacientes de nuestra muestra del tratamiento sanitario y de otros factores de resiliencia.

PALABRAS CLAVE: Resiliencia psicológica, Atentado terrorista, Trastornos por estrés postraumático. resilience more mentioned only was mentioned by ten patients of the sample, which seems to indicate a greater importance of sociofamiliar support for overcoming a traumatic experience and a smaller utility for the patients of this sample of the sanitary treatment and of other factors of resilience.

KEYWORDS: Resilience, Terrorist Assault, Stress Disorders (Post-Traumatic).

\section{Introducción:}

El 11 de marzo del 2004 sucedieron en la Comunidad de Madrid una serie de atentados terroristas, 10 bombas estallaron en tres trenes con destino a la estación de Atocha. De estos tres trenes dos de ellos procedían de la localidad de Alcalá de Henares, y el otro procedente de Guadalajara había hecho parada también en esta localidad.

Esta tragedia supuso un reto para la sanidad madrileña en general y en el Centro de Salud Mental (CSM) de Alcalá de Henares en particular, que por su situación geográfica tuvo que hacer frente una mayor demanda sanitaria en relación a los atentados.

Tras una vivencia traumática, la resiliencia, la capacidad de las personas de adaptarse, afrontar y sobreponerse a situaciones de dolor emocional, tragedias o situaciones vitales adversas, va a determinar la adaptación posterior tras la vivencia del individuo, y el si esta readaptación se produce en más o menos tiempo y de una manera sana.

Hasta el momento la mayoría de los estudios previos se han centrado en factores que facilitan esta superación de lo traumático, que resultan resilientes para la víctima, entre estos factores destacan el apoyo social, (1) principalmente el familiar, la sensación de pertenencia a un grupo (2) -dando un lugar a las víctimas un reconocimiento y un apoyo que va a facilitar la superación de los hechos traumáticos, a partir de la cohesión grupal y la formación de una identidad grupal común- y el darle un sentido a la experiencia e integrarla en una visión personal, que no tiene que estar guiada por la lógica o por la consecuencia de los hechos, es una creación propia del individuo, que en ocasiones solo él comparte, pero que le ayuda a la superación del trauma (3).

Otros factores facilitadores de la resiliencia son el optimismo (3), la flexibilidad cognitiva (4) y la aceptación de lo vivido. 
ORIGINALES Y REVISIONES

La resiliencia en el contexto de atentados terroristas se ha estudiado en los últimos años, encontrándose que los individuos con mayores capacidades resilientes eran los que tenían mayor apoyo social (5), los que no tenían experiencias traumáticas previas (6) y los que utilizaban un estilo de afrontamiento activo (7). En otros estudios tras atentados terroristas el concepto de resiliencia y su asociación a la superación de lo traumático se han visto asociada a un menor uso de servicios sanitarios (8) y ausencia de uso de tóxicos tras la vivencia traumática (9).

Resumiendo hallazgos de estudios previos tras atentados terroristas se puede decir que las capacidades de resiliencia están muy relacionadas con las habilidades de afrontamiento de la víctima, pero que estas estarán a su vez mediadas por el entorno social y familiar así como por el significado que el individuo y su entorno le den a la experiencia vivida. $(4,7,8,9)$

Es decir, ante una situación traumática cada individuo pondrá una serie de recursos (factores de resiliencia) en juego para lograr la superación de lo vivido de la manera más sana y readaptarse a su vida previa, pero a su vez estos recursos estarán mediados por características del individuo, de su entorno sociofamiliar y del contexto de lo traumático, que actuaran como facilitadores o dificultaran la superación de lo vivido a partir de cómo median sobre estas capacidades resilientes (4).

El objetivo de este estudio es el conocer los factores de resiliencia que los pacientes que acudieron al CSM con un motivo de consulta en relación a los atentados terroristas consideraban más les habían ayudado a superar la experiencia traumática y tratar de relacionar dichos factores de resiliencia con otros factores ya sean individuales, sociales o del tratamiento recibido durante el tiempo de intervención en el CSM. Finalmente se trata de relacionar los resultados con los conocimientos existentes sobre resiliencia y aprendizaje postraumático tras situaciones traumáticas.

\section{Material y Método:}

El estudio fue llevado de manera retrospectiva, naturalística, a partir del estudio de las historias clínicas del centro de salud. El periodo de inclusión fue desde el 12 de marzo de 2004 (un día después de los atentados) hasta el 30 de junio de 2004, consideramos este periodo de tiempo suficiente como para que los pacientes que tuviesen sintomatología clínica hubiesen acudido a consulta. Durante este periodo de tiempo se revisaron todas las historias clínicas de los pacientes que habían acudido al centro con un motivo de consulta en relación al $11 \mathrm{M}$ y que eran fáciles de identificar porque se arbitró un procedimiento especial para atenderlos. Se consideró que lo más adecuado no era sólo revisar historias nuevas abiertas durante este tiempo, sino también de pacientes con historia antigua, que habían sido dados de 
alta, y que volvieron a acudir por este motivo durante este tiempo. El criterio para la inclusión fue el de demandar ayuda en relación con los atentados, se incluyeron tanto pacientes que habían estado en los trenes siniestrados el $11 \mathrm{M}$, aquellos que habían perdido a familiares y amigos o éstos habían resultado afectados y algunos que no habían perdido a nadie durante los mismos, ni se encontraban allí, pero relacionaban su clínica con dichos atentados. El estudió fue llevado únicamente con población adulta, no se incluyeron menores de 18 años. Las características sociodemográficas de la muestra se muestran de manera resumida en la tabla 1.

Tabla 1

Variables sociodemográficas

\begin{tabular}{|c|c|c|}
\hline VARIABLE DEMOGRÁFICA & NÚMERO DE PACIENTES & PORCENTAJE \\
\hline \multicolumn{3}{|l|}{ Sexo } \\
\hline Masculino & 37 & $35.57 \%$ \\
\hline Femenino & 67 & $64.43 \%$ \\
\hline \multicolumn{3}{|l|}{ Nacionalidad } \\
\hline Española & 56 & $54 \%$ \\
\hline Este de Europa & 23 & $22 \%$ \\
\hline América latina & 21 & $20 \%$ \\
\hline África subsahariana & 2 & $1.9 \%$ \\
\hline Norte de Africa & 1 & $0.96 \%$ \\
\hline \multicolumn{3}{|l|}{ Estado Civil } \\
\hline Soltero & 42 & $40 \%$ \\
\hline Casado & 47 & $35 \%$ \\
\hline Pareja de hecho & 6 & $5.7 \%$ \\
\hline Separado-divorciado & 6 & $5.7 \%$ \\
\hline Viudo & 3 & $2.7 \%$ \\
\hline \multicolumn{3}{|l|}{ Convivencia } \\
\hline Solo & 9 & $8.6 \%$ \\
\hline Familia de origen & 28 & $27 \%$ \\
\hline Familia propia & 52 & $50 \%$ \\
\hline Otras familias & 3 & $2.8 \%$ \\
\hline Otro tipo de convivencia & 11 & $10.51 \%$ \\
\hline \multicolumn{3}{|l|}{ Nivel de estudios } \\
\hline Licenciado-diplomado-doctor & 27 & $25.96 \%$ \\
\hline COU-Bachiller & 38 & $36.5 \%$ \\
\hline Graduado escolar & 29 & $27.88 \%$ \\
\hline Sin estudios & 10 & $9.6 \%$ \\
\hline \multicolumn{3}{|l|}{ Nivel sociolaboral } \\
\hline Medio & 23 & $25 \%$ \\
\hline Medio-bajo & 45 & $48.9 \%$ \\
\hline Bajo-muy bajo & 12 & $13 \%$ \\
\hline
\end{tabular}


ORIGINALES Y REVISIONES

A los 2 años y medio de las atentados (entre enero y febrero del 2007) se contactó de nuevo con los pacientes de manera telefónica, se les explicó que se estaba realizando un estudio sobre los atentados del $11 \mathrm{M}$ con afectados en ellos, y tras resolver dudas en relación a ello se le preguntaba "Qué es lo que cree que más le ha ayudado a superar la situación traumática vivida", además de preguntárseles su valoración sobre los servicios sanitarios recibidos y sobre la superación de la experiencia traumática.

La encuesta telefónica constaba de 5 preguntas (anexo 1).

En la entrevista telefónica se recogía literalmente lo que los pacientes contestaban, para poder elaborar posteriormente la lista de categorías y de etiquetas a partir del discurso de los pacientes.

Para el análisis de los datos se aplicaron estadísticos descriptivos utilizando para ello el programa estadístico SPSS v14.0

\section{Resultados:}

Se contabilizaron en el estudio 104 historias que incluían el criterio de inclusión (sintomatología o motivo de consulta en relación al 11M). Para el estudio de las variables sociodemográficas se consideró como válida la hoja de filiación, documento estadístico que recoge el centro, a través de los administrativos, durante la primera cita, previa a la evaluación del terapeuta. El teléfono para contactar con los pacientes se consiguió a través de esta hoja de filiación.

A través de las respuestas dadas por los pacientes en la entrevista telefónica se trató de realizar un análisis del discurso, de forma que se puedan clasificar en etiquetas comunes que agrupen las respuestas dadas por los pacientes en relación a los factores de resiliencia que consideraban más les habían ayudado a la superación de la situación vivida ( factores de resiliencia), es decir se han elaborado a partir de las respuestas de los individuos constructos, que puedan servir para el estudio de lo que esta muestra considera desde su punto de vista (subjetivo por tanto) que han sido los factores de resiliencia, lo que más facilitó su readaptación tras la experiencia traumática.

La pregunta se realiza por tanto de una forma abierta, de manera que los pacientes podían responder cuantas respuestas quisiesen, si bien en este estudio se ha optado en incluir únicamente la primera de las respuestas dadas por los encuestados.

Las categorías de factores de resiliencia que se elaboraron a partir de las respuestas de los pacientes y el número de pacientes que las mencionaron fueron las siguientes: 
Factores asociados al entorno sociofamiliar: Muchos de los pacientes consideran que lo que más les ha ayudado a superar la situación traumática tiene que ver con el apoyo de la gente cercana, principalmente de su familia, pero también de amigos o familia. Dentro de esta categoría se incluyeron repuestas como familia, pareja, hijos, padres, pero también amigos, vecinos u otros grados de parentesco. Destaca que es el factor de resiliencia más mencionado por la muestra con diferencia (34 respuestas, $44.73 \%$ del total), a pesar de que el constructo social de la muestra era heterogéneo en cuanto a nacionalidad, estando conformada por un $46 \%$ de extranjeros (9). Dentro de esta categoría se incluye el apoyo familiar (9 respuestas de pacientes), el de la pareja (3 respuestas), amigos (6 respuestas), hijos (4 respuestas), gente cercana (1 respuestas) y vecinos (1 respuestas).

Dentro de cada una de las subcategorías la respuesta más mencionada, más de la mitad de los pacientes es la de la familia, a nivel global y sin especificar, mientras que también han sido mencionadas otras respuestas que quedarían englobadas dentro de familia, pero que fueron respondidas explícitamente, como son hijos, y si se quiere incluir la pareja. Cabe destacar que de la misma manera que éstos se han incluido, nadie respondió los padres, como factor de resiliencia, a pesar de que algunos de los afectados eran gente joven, o que incluso vivía con los padres, aunque es de suponer que ese apoyo esté incluido en las respuestas del apoyo de la familia

Factores asociados al tratamiento: Incluye todas las respuestas en las que lo que los encuestados consideran que lo que más les ha ayudado a la superación de lo vivido tiene que ver con el tratamiento que recibieron en el CSM u otros dispositivos del área. En esta categoría se incluyeron respuestas como la ayuda de los profesionales (psiquiatras y/o psicólogos), el tratamiento farmacológico recibido o el tratamiento psicoterapeútico, que incluye la modalidad grupal, a partir de grupos psicoterapéuticos realizados en el Hospital de Día de área con afectados de los atentados.

Los factores asociados al tratamiento son mencionados por 10 pacientes de la muestra (13.15\% del global), siendo el segundo grupo de respuestas más mencionado.

Factores asociados a la respuesta individual al trauma: Dentro de esta categoría se ha incluido a todas aquellas respuestas que han dado los pacientes que tiene que ver con lo que cada uno de ellos hizo a nivel individual para poder superar la situación traumática, ya sean conductas o respuestas mentales. Engloba las actitudes, respuestas y cambios que los individuos desarrollaron de manera individual y autónoma para poder superar lo vivido. Esto incluye un amplio grupo de respuestas, ya que son todas aquellas cuestiones que los sujetos traumatizados hicieron a nivel individual, ya sea el tratar de no pensar, el olvidar (si bien aceptamos que puede no ser un acto al menos voluntario), o conductas (irse de Alcalá), visitar el 
ORIGINALES Y REVISIONES

monumento a las víctima, no pensar, mirar hacia delante, no ir al Centro de Salud Mental, tratar de olvidar, reflexionar, la necesidad de adaptarse, el autocontrol o incluso la sugestión. Es el grupo de respuestas más heterogéneo y el mencionado en tercer orden por la muestra (8 pacientes $10.52 \%$ del global). Aquí están englobadas respuestas como no pensar (respondido por 3 pacientes), mirar hacia delante, hacia el futuro (2 respuestas), tratar de olvidar, irse fuera de Alcalá y reflexionar (una respuesta).

Factores asociados a las características propias del individuo: Engloba las respuestas que tienen que ver con ver con los rasgos distintivos para el sujeto que tiene que ver con cómo es él y que le han ayudado a superar la situación traumática. Dentro se incluyeron respuestas como mi carácter o mi fuerza de voluntad pero también la edad. Son por tanto respuestas en las que el individuo piensa que lo que más le ayudó a superar la situación vivida son aspectos propios suyos que pudo utilizar en ése momento. Es el cuarto factor de resiliencia mencionado por la muestra (7 pacientes, 9.21\% del global), incluyéndose respuestas como la edad (una respuesta), las características del individuo, que se usan como recursos propios ante la situación traumática, como serían el carácter (tres respuestas), o la fuerza de voluntad (tres respuestas).

Factores asociados a la recuperación de la vida previa al trauma: Es mencionado por 4 pacientes (5.26\% de la muestra), incluye las respuestas como el poder volver a la rutina (dos respuestas), el paso del tiempo o el vivir el día a día (una respuesta cada una).

Factores asociados a las relaciones interpersonales: Es mencionado por cuatro pacientes $(5.26 \%$ de la muestra). Dentro de esta categoría se encuentran las respuestas, el poder hablar sobre lo sucedido, sentirse comprendida, sentirse escuchada y conocer a gente en una situación similar (una respuesta cada una). Este ítem incluye todos aquellos factores que tiene que ver con lo relacional, con las relaciones humanas, quizás se corresponda con lo que le ha resultado de utilidad a los individuos del apoyo sociofamiliar, pero en mayor profundidad o concreción. Lógicamente esta categoría tiene que ver también con los factores asociados al entorno sociofamiliar, pero entendemos por un lado que dentro de esta respuesta se incluyen otros aspectos que tienen que ver con esa relación de apoyo y que por otro lado las respuestas asociadas a las relaciones interpersonales suponen que eso (sentirse escuchada...), sucede en varias relaciones de apoyo ( y no únicamente en la familiar) y que por eso es por lo que el paciente cree que es lo que más le ha ayudado.

Factores asociados a la espiritualidad y la religión: Incluye las respuestas de los pacientes que piensan que lo que más les ayudó a la superación de lo traumático tiene que ver con aspectos asociados a creencias o prácticas religiosas, sin entrar a determinar si es la católica, musulmana o de otro tipo (si bien por el constructo 
de la población atendida en su mayor parte sería la católica, al estar la muestra formada principalmente por españoles, sudamericanos y rumanos). Es mencionada por tres pacientes (3.94\% de la muestra). Incluye también respuestas que tienen que ver con factores asociados a la espiritualidad del individuo. En cuanto a estos factores asociados a la espiritualidad tendría que ver con cuestiones no tangibles pero que el individuo considera que le han sido útiles para la superación de la situación traumática, ya sea la fe en algo concreto, el pensar que hay otra vida, o el uso de técnicas de meditación, autoconocimiento o autoayuda para la superación del hecho traumático, si bien hay que destacar que todas las respuestas englobadas aquí incluyeron a Dios (tres respuestas).

Trabajo: Incluye las respuestas en las que lo que ayudó al paciente tiene que ver con aspectos asociados a su ocupación laboral, ya sea el poder volver a incorporarse o cumplir su horario.

Fue mencionado por tres pacientes (3.94\% de la muestra).

Factores asociados a las consecuencias del trauma: Dentro de esta categoría se ha incluido aquellas respuestas, en las que el haber podido superar la situación traumática tiene que ver con las consecuencias que a nivel sintomático ha tenido para el individuo lo vivido. Dentro de esta categoría están incluidos las respuestas estar bien físicamente (no tener secuelas físicas), o bien el mejorar en determinados síntomas psicológicos que han sido originados por la vivencia traumática, por ejemplo el empezar a dormir bien después de lo sucedido (respondido por un paciente cada una). Es mencionado por dos pacientes (2.6\% de la muestra).

Factores en relación a las experiencias propias del individuo: Es mencionado por un paciente ( $1.31 \%$ de la muestra). Incluye las respuestas en las que el individuo considera que lo que más le ayudó a superar la situación traumática tiene que ver con experiencias previas, generalmente de tipo traumático también. A pesar de ser una de las más escasamente mencionadas se ha decidió incluirla por la importancia de las experiencias traumáticas previas para tener que hacer frente el individuo en el futuro a otra situación traumática (lo que tiene que ver con la resiliencia).

La tabla 2 muestra los resultados en cuanto a los factores de resiliencia que se acaban de comentar:

\section{Discusión:}

Las respuestas sobre los factores de resiliencia de esta muestra resaltan en primer lugar la importancia del apoyo familiar (y en menor medida social), para la superación de experiencias traumáticas, acorde con los resultados de la mayoría de 
ORIGINALES Y REVISIONES

Tabla 2

Factores de resiliencia mencionados por los pacientes de la muestra.

\begin{tabular}{l|c|c}
\hline \multicolumn{1}{c|}{ CATEGORÍA } & NÚMERO DE RESPUESTAS & PORCENTAJE DEL TOTAL \\
\hline $\begin{array}{l}\text { Factores asociados al entorno } \\
\text { sociofamiliar. }\end{array}$ & 34 & $44.73 \%$ \\
\hline Factores asociados al tratamiento & 10 & $13.15 \%$ \\
\hline $\begin{array}{l}\text { Factores asociados a la espiritualidad } \\
\text { y religión }\end{array}$ & 3 & $3.94 \%$ \\
\hline $\begin{array}{l}\text { Factores asociados a las relaciones } \\
\text { interpersonales }\end{array}$ & 4 & $5.26 \%$ \\
\hline $\begin{array}{l}\text { Factores asociados a las características } \\
\text { propias del individuo }\end{array}$ & 7 & $9.21 \%$ \\
\hline $\begin{array}{l}\text { Factores asociados a las consecuencias } \\
\text { físicas y psicológicas del trauma }\end{array}$ & 2 & $2.61 \%$ \\
\hline $\begin{array}{l}\text { Factores asociados a la respuesta } \\
\text { individual al trauma }\end{array}$ & 8 & $10.52 \%$ \\
\hline $\begin{array}{l}\text { Factores asociados al pasado y a las } \\
\text { experiencias del individuo }\end{array}$ & 1 & $1.31 \%$ \\
\hline $\begin{array}{l}\text { Trabajo } \\
\begin{array}{l}\text { Factores asociados a la recuperación } \\
\text { de la vida previa al trauma }\end{array}\end{array}$ & 3 & $3.94 \%$ \\
\hline
\end{tabular}

los estudios sobre experiencia traumática, si bien su importancia se ha estudiado en casi todas los trastornos mentales $(10,11)$. Destacado es también el apoyo de la red social, y más de los amigos, seguramente relacionado con el alto número de inmigrantes (46\% de la muestra), algunos de los cuales con escasa red familiar en España y el papel que tiene para ellos los amigos, también como factores de apoyo se incluyen la gente cercana y los vecinos. No cabe duda que para la mayoría de los pacientes que sufren una enfermedad mental, o que han pasado por una situación traumática como puede ser ésta, el apoyo de la gente cercana y de la familia es uno de los factores más importantes para poder superar su situación según se ha visto en estudios previos (12), en el caso de aquellos que no tengan ese apoyo familiar, se centrarán sobre todo en el apoyo social (13). Estos resultados coinciden con los realizados tras situaciones traumáticas, que dan un papel destacado al apoyo sociofamiliar para su superación $(4,5,12,13)$.

El segundo grupo o categoría más respondido son los factores asociados al tratamiento, entre los que se incluyen las respuestas de los que dijeron que lo que más les ayudó fueron los profesionales sanitarios (psiquiatras y psicólogos), los fármacos y los grupos psicoterapéuticos. Una primera aclaración es que no todos los pacientes fueron derivados a los grupos psicoterapéuticos del Hospital de Día (que funcionó como una modalidad terapeútica diferente), y que no todos los pacientes recibieron o aceptaron medicación. Destaca el valor reducido dentro del 
global de respuestas de los factores asociados al tratamiento. Esto hace plantearse el posible papel reducido de la ayuda sanitaria ante las situaciones traumáticas, y sobre todo la escasa percepción positiva para los afectados del tratamiento recibido como factor de resiliencia. Ante esto hay que replantearse si el papel de los profesionales podría realizarse de otra manera, o habría aspectos mejorables. No queda muy claro, si para los pacientes lo recibido era lo que esperaban y les resultó útil, pero a tenor de las respuestas no fue lo fundamental para la readaptación tras la experiencia traumática. Con ello podemos hipotetizar que aunque para los pacientes de la muestra la ayuda sanitaria y profesional fue adecuada no fue lo más importante para la readaptación tras la situación traumática, sino que en todo caso facilitó que los pacientes pudiesen recibir otro apoyo que les resultó más importante como factor de resiliencia (el familiar), o colocarles en una posición desde la que les resultó de más ayuda, pero que aunque es un factor que la mayoría considera que fue útil y cumplió sus expectativas no es lo más importante para la mayoría de los pacientes de la muestra como factor que de resiliencia.

En cualquier caso, el hecho de que el papel como factor de resiliencia de los profesionales y los tratamientos aplicados sea relativo coincide con los estudios que abogan por un papel menor de los profesionales ante situaciones traumáticas $(14,15,16)$, dado que la mayoría de las personas no necesitan ni consultar ante estas vivencias, y esto puede explicar porque la mayoría de los pacientes de la muestra a los dos años y medio ya no acudían al CSM.

El tercer grupo de respuestas más contestado es el de las respuestas individuales al trauma, se incluyen aquí un grupo de respuestas heterogéneo que tienen en común que son respuestas que el individuo desarrolla específicamente ante la situación traumática, con el objetivo específico de poderlo superar, como reacción de coping.

Destaca que la mayoría de las acciones de estos pacientes que consideran que les ayudaron podríamos definirlas como más cercanas a las conductas evitativas de coping (no pensar, tratar de olvidar, irse de Alcalá, no ir al CSM), y muy pocas como cercanas al afrontamiento de la situación traumática (reflexionar, mirar hacia delante), si bien estas respuestas evitativas se han asociado con peor calidad de vida percibida tras una situación traumática (17). En contra de lo que pudiera parecer, la mayoría de los pacientes lo que creen que les ha ayudado es el tratar de huir o de evitar estímulos en relación al trauma vivido, y resulta más curioso que consideren que esto les ha ayudado, cuando en muchas ocasiones en terapia se trabaja en el sentido contrario, como forma de superación de lo traumático, tratando de que no se produzcan respuestas evitativas, que en ocasiones acaban enquistando situaciones traumáticas, dificultando la normalización de la vida. Quizás es con esto con lo que tenga que ver que algunos pacientes dejasen de acudir porque pensaban que no les beneficiase o les hacía daño, el ir al CSM (los profesionales trabajarían segura- 
ORIGINALES Y REVISIONES

mente hacia las respuestas de afrontamiento y no evitativas, cuando a los pacientes lo que pensaban que les ayudaba eran estas respuestas de evitación y lógicamente sufrían al acudir al CSM).

Se observa que muchos de los pacientes tras la situación traumática tienden a huir de cualquier pensamiento, situación o lugar que les recuerde al trauma, lo que está incluido como síntomas evitativos en el TPET, este tipo de respuestas superan en número a las que podríamos llamar de afrontamiento activo: Reflexionar, mirar hacia delante, y faltaría saber cuáles son más eficaces a medio plazo para la superación de la situación traumática, aunque en los estudios previos el afrontamiento activo se considera como un factor de protección, así como que facilita la existencia de cauces para la expresión emocional $(15,16)$, y las otras se asocian a peor calidad de vida percibida tras una vivencia traumática (17).

El cuarto grupo de respuestas más contestado es el de factores asociados a las características propias del individuo, rasgos o cualidades del individuo que o bien están presentes previamente antes de la situación traumática, o salen a la luz tras la misma, y que el individuo usa como recursos propios para la superación de la situación traumática. Recursos que puede de no haber sido por el trauma vivido tal vez no hubiesen salido a la luz, pero que el individuo considera como propios, y que le han ayudado ante lo traumático. En este contexto los rasgos caracteriales que se han asociado a una mejor evolución en estudios previos serán la sociabilidad, la extroversión y el bajo neuroticismo (18), además de un adecuado autoconcepto, de la misma manera se ha encontrado la edad adulta un factor de protección del mismo modo que resulta un factor de riesgo la edad infantil (18).

El quinto grupo de respuestas más nombrado sería el de factores asociados a la recuperación de la vida previa al trauma. En relación a las respuesta de la encuesta, para los pacientes una de las consecuencias que ellos mismos consideran que les impiden superar la situación traumática es el haber perdido aspectos propios de su carácter o de su vida, por ello, también considerarán que los factores asociados a que recuperen su rutina y su ritmo de actividades previos a la situación traumática estará entre lo que crean que más les ha ayudado. Habría que preguntarse qué es lo que les ha ayudado hasta que esto se ha podido producir, pero parece que para algunos pacientes es vital el poder hacer las mismas cosas que hacían antes de los atentados, tanto a nivel conductual como cognitivo. En este sentido es importante el papel de los medios de comunicación, a los que en muchas ocasiones se les achaca que no permiten que los pacientes hagan precisamente esto, recuperar la vida previa qué tenían (19). Se ha estudiado la importancia de la recuperación de la vida previa al trauma como factor de buen pronóstico ante una situación traumática $(20,21)$, por tanto que el paciente tenga la sensación de estar recuperando aspectos de su vida que en este caso los atentados le habían arrebatado se puede considerar como algo resiliente, que facilita la superación de lo traumático, como algunos 
ORIGINALES Y REVISIONES

estudios han mostrado (22), del mismo modo que la pérdida o no recuperación se puede considerar un factor de riesgo $(20,21)$.

El sexto grupo más respondido es el de factores asociados a las relaciones interpersonales. Este grupo engloba a respuestas de pacientes que lo que consideran que les ha ayudado tiene que ver con situaciones que se dan en las relaciones humanas. Esto enlazaría con la necesidad ante una situación traumática de hablar sobre ello, y de aspectos reforzadores que se puedan dar en una relación familiar, pero también con un amigo o en una relación terapeútica. Esto no quiere decir que para todos los pacientes sea necesario hablar sobre el trauma vivido, pero sí con la sensación de que si lo hacen después de lo ocurrido pueden ser escuchados y comprendidos, cuando a ellos les resulta a veces difícil entender el porqué ha sucedido lo que ha pasado. Así mismo iría en contra de la aparición de respuestas emocionales que podrían perpetuar el trauma, como son la culpa, la vergüenza...(22).

Por otro lado la respuesta conocer gente en una situación similar estaría en relación con lo que se consideran los factores terapéuticos comunes de los grupos (22), en este caso la universalidad, saber que después de lo ocurrido no se es único, sino que hay gente que ha vivido lo mismo, y que tiene pensamientos y sentimientos parecidos a los que puede tener uno, aunque al sujeto le resulten extraños o le generen sufrimiento el tenerlos (en este caso cumpliría una función normalizadora). Estos factores no tienen porqué darse en una relación terapeútica. Sobre esta temática los estudios coinciden en que es un factor positivo cara la superación de lo traumático, y que la existencia de cauces para canalizar la expresión emocional es un factor de protección ante lo traumático, en cuanto a que sentirse escuchado, comprendido, aceptado facilitan que se pueda resolver correctamente lo traumático $(16,22)$, elementos estos que solo se dan en relaciones interpersonales (15), con lo que esto enlazaría también con la importancia de las relaciones sociales y el apoyo sociofamiliar para la superación de lo traumático (18).

La séptima categoría en número de respuestas son los factores asociados a la espiritualidad y la religión. Faltaría saber qué es lo que realmente hay detrás de lo estas respuestas, si es el poder encontrar respuestas a lo sucedido o bien esperanza, o valor para soportar el sufrimiento. Aunque es conocida la importancia de la religión para la superación de patologías psiquiátricas así como situaciones adversas, principalmente en el sentido de encontrar un significado a lo sucedido $(23,24)$ en esta muestra no son los factores de resiliencia más mencionados, si bien fueron mencionados por 3 pacientes, un porcentaje seguramente inferior al del porcentaje de creyentes en el caso de que lo hubiéramos preguntado. Esto parece indicar un menor valor a la religión y la espiritualidad tras una vivencia traumática en esta muestra, si bien hay que destacar que todos los pacientes que lo mencionaban consideraban que para ellos había sido vital.

El octavo grupo de respuestas sobre factores de resiliencia más mencionado 
ORIGINALES Y REVISIONES

son los factores asociados al trabajo, para estos pacientes el trabajo fue un factor que creen que les ayudó a la superación de la experiencia traumática, ya sea como forma de normalización o de recuperación de su vida previa (16), por las relaciones interpersonales que allí establecen $(10,25,26)$, porque hacía que durante unas horas al día estuviesen centrados en algo que no tenía que ver con lo vivido...Esto estaría en relación con las habilidades de afrontamiento de cada paciente, y con la normalización a través de la recuperación de la rutina de la vida previa. Además el volver al trabajo indica para muchos, que iban en los trenes al trabajo, el poder volver a hacer la misma rutina cotidiana que hacían antes de los atentados, entre lo que estaba el poder volver a coger un transporte público.

El noveno grupo de respuestas es el de respuestas asociadas a las consecuencias psicológicas del trauma. Esto llevaría detrás algo así como un ejercicio de evaluar de una manera realista lo que se ha perdido, como el trabajo que en ocasiones se hace en los duelos, y la ayuda que supone el pensar que las consecuencias podían haber sido mucho peores. También los estudios hablan de la importancia tras una vivencia traumática de la situación física en la que ha quedado el paciente, así como de la importancia de la ausencia de secuelas para poder readaptarse de una manera adecuada $(17,27)$. Este grupo de respuestas es la que en parte se asemeja más a una mejoría clínica, desaparición de síntomas asociados a la vivencia traumática, lo que nos hace plantearnos si realmente lo más adecuado tras una vivencia traumática es valorar la situación clínica según criterios DSM o CIE, cuando en realidad en esta muestra son pocos los que consideran que su mejoría tenga que ver con la desaparición de síntomas clínicos asociados a la vivencia traumática, sin embargo a nivel clínico estos son de los factores que más se relacionan con una mejoría sintomática.

Finalmente el décimo grupo incluye las experiencias vividas previamente (una respuesta), esto englobaría el papel de las situaciones traumáticas vividas previamente, así como que el aprendizaje postraumático puede ayudar al afrontamiento y superación de experiencias traumáticas que pudiesen aparecer posteriormente. Lógicamente no es muy habitual que haya individuos, y quizás menos en España que en su vida tengan que afrontar dos situaciones traumáticas diferentes, por lo que es una respuesta que solamente dio un individuo. Esto iría a favor del factor protector de las experiencias traumáticas bien resueltas en el pasado cara al afrontamiento de nuevas situaciones traumáticas, aunque de no ser esto el vivir otra situación traumática puede hacer resurgir traumas previos no resueltos. Los estudios al respecto no acaban de encontrar si el haber sufrido experiencias traumáticas previas, ante la aparición de otra situación traumática, resultan protectoras (8), o de riesgo (13), aunque seguramente esto esté a su vez marcado por capacidades resilientes del individuo y del aprendizaje postraumático. En los estudios sobre atentados del $11 \mathrm{~S}$ el haber pasado por experiencias traumáticas previas sí se aso- 
ciaba a peor evolución en cuanto a mayor riesgo de TPET y mayor uso de servicios sanitarios y medicación $(7,28,29)$.

Limitaciones del estudio: Destacamos aquí algunas características relativas a la muestra, recogida en un área urbana concreta del noreste de Madrid, la población Alcalá de Henares (como cualquier otra localidad con unas características sociodemográficas concretas), con lo que esto pueda implicar de cara a extrapolar los resultados a la población general. También el contexto del atentado (primera hora de la mañana de un día laborable en un medio de trasporte público), determina que dicha muestra sea de un nivel socioeconómico determinado, con la dificultad que implica para la extrapolación de los resultados a la población general.

Otro factor a tener en cuenta tiene que ver con el elevado porcentaje de población extranjera (46\%), que también puede dificultar la extrapolación de resultados respecto a los factores de resiliencia que se utilizan tras una vivencia traumática por lo heterogéneo que resulta.

Entendemos que el propio diseño del estudio, a través de una pregunta abierta puede suponer una limitación sobre todo en cuanto a la fiabilidad estadística de los resultados.

Finalmente el reducido tamaño muestral, y el hecho de que el estudio haya sido realizado en una muestra que acudió a los Servicios de Salud Mental pueden suponer también limitaciones. En el segundo caso porque una situación traumática del calibre del $11 \mathrm{M}$ resulta traumática para toda la población que resultó afectada de manera directa o indirecta (no únicamente para los que consultaron con los Servicios de Salud Mental), con lo que el hecho de que se trate de una muestra de población atendida en los Servicios de Salud Mental puede suponer una limitación.

Todo ello indica que futuros estudios que se quieran realizar analizando los factores de resiliencia tras una vivencia traumática deberán valorar por un lado el diseño del estudio (quizás utilizando preguntas cerradas en la que los pacientes elegirían entre una lista de factores de resiliencia cerrada, en la que podrían estar presentes algunos de los factores de resiliencia mencionados por la muestra de este estudio) y también valorar el incluir en la muestra a pacientes afectados por dicha vivencia que no hubiesen consultado con los Servicios de Salud Mental.

Finalmente se necesitaría un mayor número de estudios con tamaños muestrales más elevados para poder confirmar por un lado y extrapolar con mayor rigurosidad estos hallazgos relativos a los factores de resiliencia tras una vivencia traumática a la población general. 
ORIGINALES Y REVISIONES

\section{Conclusiones:}

El objetivo de este estudio era el determinar para una muestra de pacientes atendidos en el CSM de Alcalá de Henares tras una situación traumática, en este caso los atentados del 11M en Madrid lo que los pacientes consideraban les había resultados más útil para superar de una manera sana la experiencia traumática.

El factor de resiliencia más mencionado era el entorno sociofamiliar. Esto recalca la importancia de la red sociofamiliar ante las enfermedades mentales en general y las situaciones traumáticas en particular como se encuentra reflejado en gran variedad de estudios.

La segunda variable más mencionada son las asociadas al tratamiento, mencionada por 10 pacientes (13.15\% de la muestra) un porcentaje escaso del global, lo que puede indicar un papel menor de la ayuda sanitaria profesional ante una vivencia traumática, si bien es la segunda más respondida.

A partir de ahí son mencionadas otros factores de resiliencia en menor medida, que tienen que ver con factores asociados a la respuesta individual al trauma (8 respuestas), los factores asociados a las características propias del individuo (7 respuestas) o a la recuperación de la vida previa al trauma (4 respuestas). Otros factores de resiliencia aparecieron de manera escasa en las respuestas de los pacientes como el trabajo, las vivencias propias del individuo o la espiritualidad y la religión.

Nuestros resultados en cuanto a los factores de resiliencia ante una situación traumática coinciden con estudios previos que resaltan la importancia vital de los factores sociofamiliares ante este tipo de vivencias.

\section{BIBLIOGRAFÍA:}

(1) Yehuda R., McFarlane AC., Shalev AY. Predicting the development of posttraumatic stress disorder from the acute response to a traumatic event. Biol Psychiatry. 1998;44:1305-1313.

(2) FREDRICKSON BL. The role of positive emotions in positive psychology. The broadenandbuild theory of positive emotions. Am. Psychol. 2001;56:218-26.

(3) Seligman MEP. 2002. Authentic Happiness. New York: Free PTugade MM, Fredrickson BL. 2002.

(4) Bonano GA., Galea S., Vlahov D. What predicts psychological resilience after disaster? The role of demographics, resources, and life stress. J Consult Clin Psychol. 2007 Oct;75(5):671-82.

(5) Schelenger WE, CadDell JM et al. Psychological reactions to terrorists attacks. Findings from the national study of American's reaction to september 11. JAMA. 2002; 288: 581-588.

(6) NoRTh CS, Nixon SJ, SHARIAT S ET AL. Psychiatric disorders among survivors of the Oklahoma City bombing. JAMA. 1999; 282: 755-762. 
ORIGINALES Y REVISIONES

(7) Boscarino Ja, Galea S, Ahern J, Resnick H, Vladov. Psychiatric medication use among Manhattan residents following the World Trade Center disaster. J Trauma Stress. 2003 Jun; 16 (3): 301-6.

(8) Schuster MA, STEIN BD JAICOX LH ET AL. A national survey of stress reactions after september 11, 2001, terrorists attacks. N. Engl. J. Med. 2001; 345 1507-1512.

(9) Demanda e atención especializada a la salud mental por los atentados del $11 \mathrm{M}$ en Alcalá de Henares: La influencia de los factores sociodemográficos". Benito Ruzz A, Lahera Forteza G, Bentto Ruiz G, OudDa Santos L. Revista de la AEN". Noviembre 2007

(10) Breslau N, Davis GC. Posttraumatic stress disorder in an urban population of young adults: risk factors for chronicity. Am J Psychiatry. 1992;149:671-675.

(11) Kessler, RC.; Sonnega, A.; Bomet, E.; Hughes, M.; Nelson, CB.; Breslau, N. Epidemiological risk factors for trauma and PTSD. In: Yehuda R. editor. Risk factors for posttraumatic stress disorder. Washington DC, American Psychiatric Press; 1999. pp. 23-59

(12) Green BL, Grace MC, Gleser G. Identifying survivors at risk: long-term impairment following the Beverly Hills Supper Club fire. J Consult Clin Psychol. 1985;53:672-678.

(13) Vogues M, Romney D. Risk and resiliency factors in posttraumatic stress disorder. Ann. Gen.Hosp. Psychatry. 2003;2:4.

(14) Lobban F, Barrowclough C, Jones S. A review of the role of illness models in severe mental illness. Clin. Psychol. Rev. 2003; 23: 171-196.

(15) Hoge EA, Austin ED, Pollack MH. Resilience: research evidence and conceptual considerations for posttraumatic stress disorder. Depress Anxiety. 2007;24(2):139-52. Review.

(16) BonAnNo G. Loss, trauma and human resilience: have we underestimated the human capacity to thrive after extremely aversive events? American Psychologist, 2004 (59), 20-28.

(17) Ardenne P, Capuzzo N et al. Subjetive quality of life and postraumatic stress disorder. J. Ner. Men. Dis. 2005; 193 (1): 62-66.

(18) Bisson Ji, Ehlers A, Matthews R, Pilling S, Richards D, Turner S. Psychological treatments for chronic post-traumatic stress disorder. Systematic review and meta-analysis.Br J Psychiatry. 2007 Feb;190:97-104. Review

(19) Ahern J, Galea S. Television images and psychopatology symptoms after the september 11 terrorists attacks. Psychiatry. 2002, 65: 289-300.

(20) Breslau N, Chilcoat HD, Kessler RC, Davis GC. Previous exposure to trauma and PTSD effects of subsequent trauma: results from the Detroit area survey of trauma. Am J Psychiatry. 1999;156:902-907.

(21) JACELON CS. The trait and process of resilience. J Adv Nurs. 1997 Jan;25(1):123-9. Review.

(22) WAGNILD G. Resilience and successful aging. Comparison among low and high income older adults. J Gerontol Nurs. 2003 Dec;29(12):42-9.

(23) Koenig HG, George LK, Trtus P. Religion, spirituality, and health in medically ill hospitalized older patients. J. Am. Ceriatr. Soc. 2004;52:554-62.

(24) Braam AW, Beekman AT, Deeg DJ, Smit JH, van Tilburg W. Religiosity as a protective or prognostic factor of depression in later life: results from a community survey in the Netherlands. Acta Psychiatr. Scand. 1997; 96:199-205.

(25) Tedeschi RG, CALHoun LG et al. The posttraumatic growth inventory: measuring the positive legacy of trauma. Journal of Traumatic Stress, 1996, 9: 3, 454-5.

(26) Layne, C.M., Pynoos, R.S., Salzman, W.R., Arslanagi, B., Savjak, N. et al.. Trauma/grief- 
ORIGINALES Y REVISIONES

focused group psychotherapy school-based postwar intervention with traumatized Bosnian adolescents. Group Dynamics: Theory, Research and Practice. 2001, 5, 277-290.

(27) Nandi A, Galea S, Tracy M, Ahern J, Resnick H, Gershon R, Vlahov D. Job loss, unemployment, work stress, job satisfaction, and the persistence of posttraumatic stress disorder one year after the September 11 attacks. J Occup Environ Med. 2004 Oct;46(10):1057-64

(28) Galea S, Vlahov D, Resnik H et al. Trends of probable posttraumatic stress disorder in New York after the 11S terrorisms attack. Am. J. Epidemiology 2003, 158 (6); 514-524.

(29) Boscarino JA, AdAms RE, Figley CR. Mental health service use 1-year after the World Trade Center disaster: implications for mental health care. Gen Hosp Psychiatry. 2004 Sep-Oct;26(5):346-58.

Anexo 1

Cuestionario mediante entrevista telefónica:

* Se realizaron estas cinco preguntas a los tres años de los atentados mediante encuesta telefónica a cada uno de los pacientes que fueron reclutados durante el periodo de inclusión para el estudio.

1) ¿Cree que ha podido asimilar de una manera sana la experiencia vivida?

2) ¿Cree que recibió lo que debía recibir por parte de los servicios sanitarios en Salud Mental?

3) ¿Lo que recibió por parte de los Servicios de Salud Mental fue útil?

4) ¿Dejó de acudir al Centro de Salud Mental? ¿Cuál fue el motivo?

5) ¿Qué es lo que más le ayudo a superar la situación vivida? 